\title{
Instantaneous Purified Orbit: A New Tool for Analysis of Nonstationary Vibration of Rotor System
}

\author{
SHI DONGFENG ${ }^{\mathrm{a}, *}, \mathrm{QU}_{\text {LIANGSHENG }}^{\mathrm{b}}$ and $\mathrm{BAO}_{\mathrm{MING}}{ }^{\mathrm{a}}$ \\ ${ }^{a}$ Research Institute of Vibration Engineering, Nanjing University of Aeronautics and Astronautics, Nanjing 210016, \\ P.R. China; ${ }^{\mathrm{b}}$ Research Institute of Diagnostics and Cybernetics, Xian Jiaotong University, Xian 710049, P.R. China
}

(Received 15 June 1999; In final form 8 October 1999)

\begin{abstract}
In some circumstances, vibration signals of large rotating machinery possess time-varying characteristics to some extent. Traditional diagnosis methods, such as FFT spectrum and orbit diagram, are confronted with a huge challenge to deal with this problem. This work aims at studying the four intrinsic drawbacks of conventional vibration signal processing method and instantaneous purified orbit (IPO) on the basis of improved Fourier spectrum (IFS) to analyze nonstationary vibration. On account of integration, the benefits of short period Fourier transform (SPFT) and regular holospectrum, this method can intuitively reflect vibration characteristics of a rotor system by means of parameter analysis for corresponding frequency ellipses. Practical examples, such as transient vibration in run-up stages and bistable condition of rotor show that IPO is a powerful tool for diagnosis and analysis of the vibration behavior of rotor systems.
\end{abstract}

Keywords: Fault diagnosis, Time-frequency analysis, Rotor system, Nonstationary vibration

\section{INTRODUCTION}

Nowadays, condition monitoring and fault diagnosis of rotor system based on vibration signals are discussed in many papers and dissertations, such as Chen et al. (1995), Muszynska (1995), and Shi (1998). In general, vibration signals are picked up by two mutually perpendicular displacement sensors mounted in a bearing section of a rotor, and sometimes a key phase signal is measured simultaneously. Furthermore, the received signals are pre-processed in order to suppress high frequency noise and direct component content, then analog-to-digital (A/D) converted and combined to construct the orbit diagram. Finally, if the signals are fast Fourier transformed, the spectra revealing their composition in the frequency domain can be obtained.

With large rotating machinery increasingly directed towards continuous operation with high speed and heavy loads, the vibration behavior of rotor systems is more and more complex. As a

\footnotetext{
* Corresponding author. E-mail: dfshi@hotmail.com.
} 
result, traditional processing methods are confronted with a huge challenge. On account of some intrinsic drawbacks, these methods seriously affect the reliability of diagnostic results to some extent in practice. At first, conventional methods, such as timebase, orbit and fast Fourier transform (FFT) spectrum, study vibration signals either as a function of time or as a function of frequency but not both. Hence, we cannot acquaint the regulation of frequency component variation with time. As we all know, a typical goal in vibration signal processing is to find a representation in which certain attributes are made explicit. The most important and fundamental variables in signal processing are time and frequency. In many real applications it is far more useful to characterize the signal in the time and frequency domains simultaneously. Secondly, there are many factors, such as fluid excitation mentioned by Qu et al. (1993), run-up or shutdown mentioned by Genta and Delprete (1995), rotor crack mentioned by Zheng (1998) and others, could lead to nonstationary vibration in large rotating machinery. However, the ordinary FFT spectrum can only evaluate an average spectrum over a definite time period and fully loses the nonstationary characteristics of such vibration signals. Thirdly, we would obtain the amplitude spectrum as well as phase spectrum through FFT of vibration signal. Unfortunately, the latter is always overlooked in application. However, if the phase relation in vibration analysis is neglected, a considerable part of information on rotor behavior will be lost. It is well known that the phase information together with the amplitude spectrum determines the shape of original signal. Same amplitude spectrum with different phase spectrum corresponds to completely different signal forms in time domain. Hence, the trinity of amplitude, frequency and phase information is required to reveal the characteristics of vibration signal in frequency domain completely. Finally, the traditional spectrum analysis method is unable to express the relationship between the horizontal and vertical vibrations in a bearing. As we all know, in comparison with timebase in single direction of the rotor case, the shape of orbit diagram is not sensitive to the circumferential positions of two mutually perpendicular sensors. Similarly, only by using the information of two mutually perpendicular signals we can discover the actual vibration behavior of the rotor system.

In order to overcome the above defects, researchers put forward many advanced methods. Aiming at the former two respects, Qingfeng Meng, Qu (1992) and Jiang Meng (1996) introduced the Wigner distribution and short period Fourier transform (SPFT), respectively, to process nonstationary vibration signal. Owing to the interference effect of cross-term in former and the lower resolution along frequency axis of the latter, these two methods are restricted to some extent in practice. With regard to these two defects, Don Southwick (1993, 1994) developed the full spectrum and obtained some positive results in analysis of the precession direction of rotor. Qu et al. (1989) found the holospectrum method based on multisensor integration and fusion. In virtue of its comprehensive information and obvious technological significance, this new method obtains an accomplishment in condition monitoring, fault diagnosis and vibration behavior analysis of a rotor system. Recently, aiming at the characteristics of nonstationary vibration in large rotating machinery, Lee and Han (1998) and Jian Meng (1996) integrated Wigner distributions and SPFT to full spectrum respectively. The vibration is decomposed into forward and backward components. The instantaneous precession direction could be specified according to the magnitude of the above two components. However, the other characteristics of nonstationary vibration will not be discovered in large rotating machinery.

The instantaneous purified orbit (IPO) presented in this paper stems from fusion of SPFT and holospectrum theory. This method thoroughly overcomes the four drawbacks of the traditional method. According to analysis of results from transient vibration signals in run-up stages and bistable conditions of a rotor, this method that we have proposed has proved effective in analysis of nonstationary vibration in large rotating machinery. 
This paper is organized as follows. Sections 2 and 3 describe the fundamental theory and characteristics of SPFT and holospectrum, respectively. In Section 4, a new improved Fourier spectrum, accurately calculating the amplitude, frequency and phase with dichotomy is introduced. Also, the IPO method is proposed. The application examples from our laboratory as well as petroleum plant are presented in Section 5. Finally, conclusions and future research direction are envisaged in the last section.

\section{SHORT PERIOD FOURIER TRANSFORM}

As we all know, traditional methods such as timebase and FFT spectrum, study signal either in the time domain or frequency domain but not both. To solve this problem, some techniques for joint time-frequency representation for signal have been recommended by Qian and Chen (1996). It principally contains SPFT, Wigner distribution and wavelet transform.

The SPFT is a joint time-frequency analysis method by means of extending FFT to process nonstationary signals. This transform is developed initially by Gabor in 1946, and defined as

$$
S_{f}(t, \omega)=\int_{\tau} f(\tau) \bar{\gamma}(\tau-t) \mathrm{e}^{-\mathrm{j} \omega \tau} \mathrm{d} \tau
$$

and is a regular inner product which reflects the similarity between signal $f(t)$ and elementary $\gamma(t)$ $\exp (\mathrm{j} \omega t)$. The function $\gamma(t)$ usually has a short duration and thereby it is named the window function. In general, the Gabor window function and Hamming window function are always used.

Because SPFT is based on the hypothesis that the signal must be stable in the window, the resolution is relatively low. In the signal processing procedure, the selection of window length is very important. On the one hand, longer windows improve resolution along the frequency axis. On the other hand, shorter window is concerned with a higher resolution along with the time axis. At the same time, the SPFT has no cross-term in comparison with the Wigner distribution. Therefore, the SPFT possesses a great increasing perspective in engineering field.

\section{HOLOSPECTRUM THEORY}

Holospectrum method is based on multisensor integration and fusion. It integrates the amplitude, frequency and phase information of two mutually perpendicular vibration signals. The procedure of integration is very simple (see Fig. 1): the FFT spectrum of the individual vibration signal is constructed first, and its precise amplitude, frequency and initial phase of the spectra lines are determined. Then, the holospectra composed of ellipses, straight lines and circles are constructed through the combination of corresponding spectral lines from the two FFT spectra. Meanwhile, geometrical parameters of holospectra component possess obvious significance. The length of major axis $2 a$ as well as minor axis $2 b$ of the ellipse indicates the maximum and minimum vibration amplitudes of corresponding frequency components respectively in Fig. 2. In general, $\left(a^{2}+b^{2}\right)^{1 / 2}$ indicates the vibration power

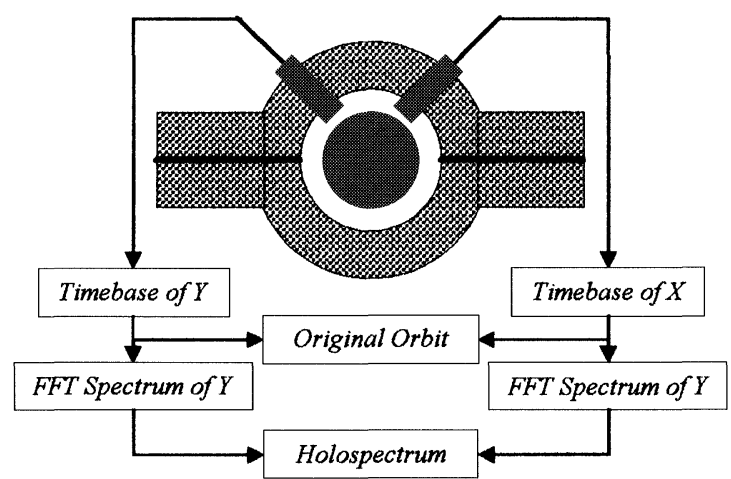

FIGURE 1 The generating procedure of holospectrum. The FFT spectrum of the individual vibration signal is constructed first, and its precise amplitude, frequency and initial phase of the spectra lines are determined, then, the holospectra are constructed through the combination of corresponding spectral lines from the two FFT spectra. 


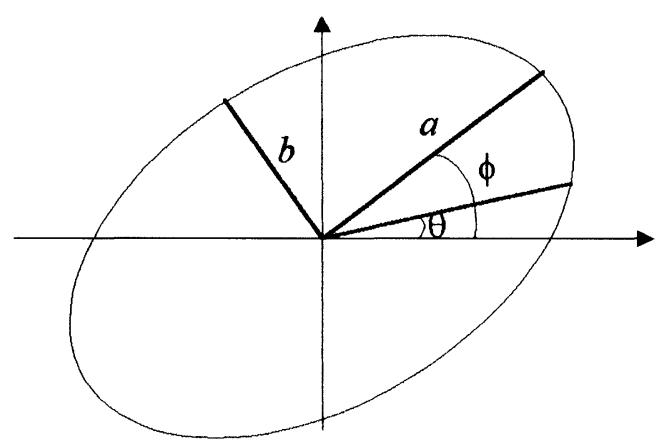

FIGURE 2 Vibration of ellipse and its parameters. The length of major axis $2 a$ as well as minor axis $2 b$ of the ellipse indicates the maximum and minimum vibration amplitudes of corresponding frequency components, respectively.

of the corresponding frequency component. The other parameters, such as the inclination angle of the major axis, $\phi$, precession direction and initial phase, $\theta$, are concerned with the vibration characteristics of the rotor system also. Instead of two ordinary, FFT amplitude spectra and two corresponding phase spectra, a single holospectrum can provide all the vibration information in one bearing. By means of the holospectrum, the amplitude information together with the phase relations of journal vibration can be shown intuitively. Obviously, in comparison with conventional methods, it is more effective to use a series of ellipses to describe the rotor vibration behavior in the space domain.

Unfortunately, holospectrum is based on ordinary FFT spectrum. If a vibration signal is nonstationary, it only presents an average effect. Consequently, it always conceals the actual vibration behavior of rotor to some extent, and sometimes even gives false results.

\section{INSTANTANEOUS PURIFIED ORBIT}

On the one hand, we can analyze the nonstationary behavior in the time domain as well as frequency domain by means of SPFT. On the other hand, we can discover the relationship between two mutually perpendicular displacements in the space domain by means of holospectrum. Inspired by the complement between SPFT and holospectrum methods, we guessed that we can obtain increasing results in the event of integration of the above two methods. By means of combining holospectrum and SPFT, we can study the vibration behavior at keeping in view the time domain, frequency domain and space domain simultaneously. According to analysis of the ellipse parameters in every time window, we can grasp the nonstationary vibration characteristics of the rotor system. However, because the time window is very short in comparison with many signals, we are forced to put forward a more effective method to acquire precise amplitude, frequency and phase information before putting this idea into effect. Hence, an improved Fourier spectrum aimed at short data is introduced.

\subsection{Improved Fourier Spectrum}

Fourier spectrum proposed by Ramirez (1985) is a powerful tool which can be used to help diagnose rotating machinery problems. Unfortunately, leakage effects appear in FFT spectra despite the use of all kinds of window functions. In fact, an FFT spectrum is the result of continuous spectrum sampled with a frequency interval $(\Delta f)$. In general, a spectrum line is not located in the center of the main-lobe, and the calculated frequency, amplitude and phase of the vibration component are not accurate. This is the so-called comb-effect (Figs. 3 and 4). The corresponding error of frequency, amplitude and phase is calculated by

$$
\begin{aligned}
& f_{\mathrm{e}}=\min (|k \Delta f-f|,|(k+1) \Delta f-f|), \\
& A_{\mathrm{e}}=x_{0} \cdot\left(W\left(f_{\mathrm{e}}\right)-1\right), \\
& \alpha_{\mathrm{e}}=\frac{\pi f_{\mathrm{e}}}{\Delta f}
\end{aligned}
$$

where $W(f)$ is the Fourier transform of window function.

It is obvious that the error of calculated frequency, amplitude and phase of vibration signal is very high. Therefore, many interpolation methods 


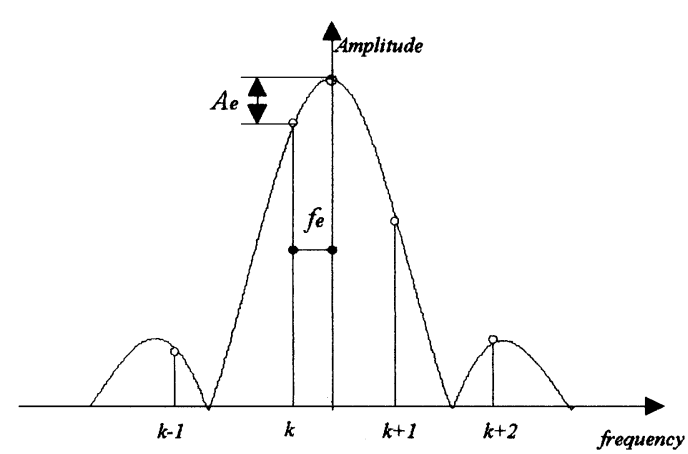

FIGURE 3 Error of frequency $\left(f_{\mathrm{e}}\right)$ and amplitude $\left(A_{\mathrm{e}}\right)$ for FFT.

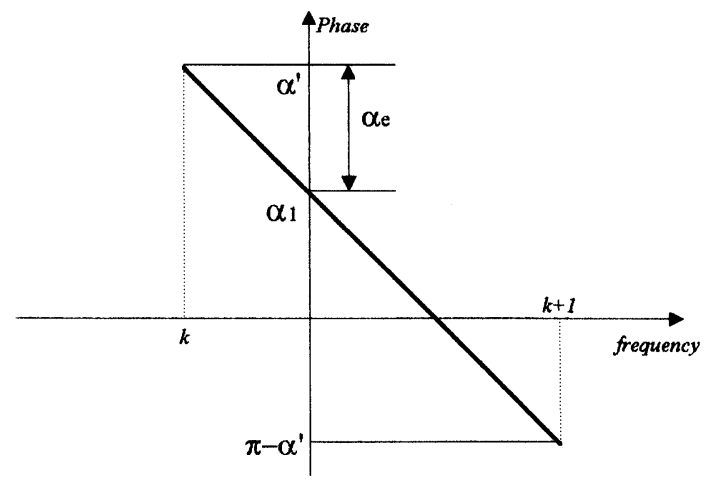

FIGURE 4 Error of phase $\left(\alpha_{\mathrm{e}}\right)$ for FFT.

are introduced to compute the precise frequency, amplitude and phase by Renders and Schoakens (1984) and Grankle (1983), respectively. Here, aiming at short data (the length is often smaller than 128 points), this paper proposes a new improved Fourier spectrum based on dichotomy to calculate above values precisely.

If we are given $N$ sample values of the signal, $x(0), x(1), \ldots, x(N-1)$, the discrete Fourier spectrum is calculated by

$$
X(k)=\frac{1}{N} \sum_{n=0}^{N-1} x(n) \mathrm{e}^{-\mathrm{j} 2 \pi n k / N} .
$$

From Eq. (5), if $k$ has a continuous value between 0 and $f_{\mathrm{s}} / 2$, and the frequency interval $\Delta f$ is arbitrarily small, the discrete spectrum lines should change into a continuous curve. Accordingly, Eq. (5) turns

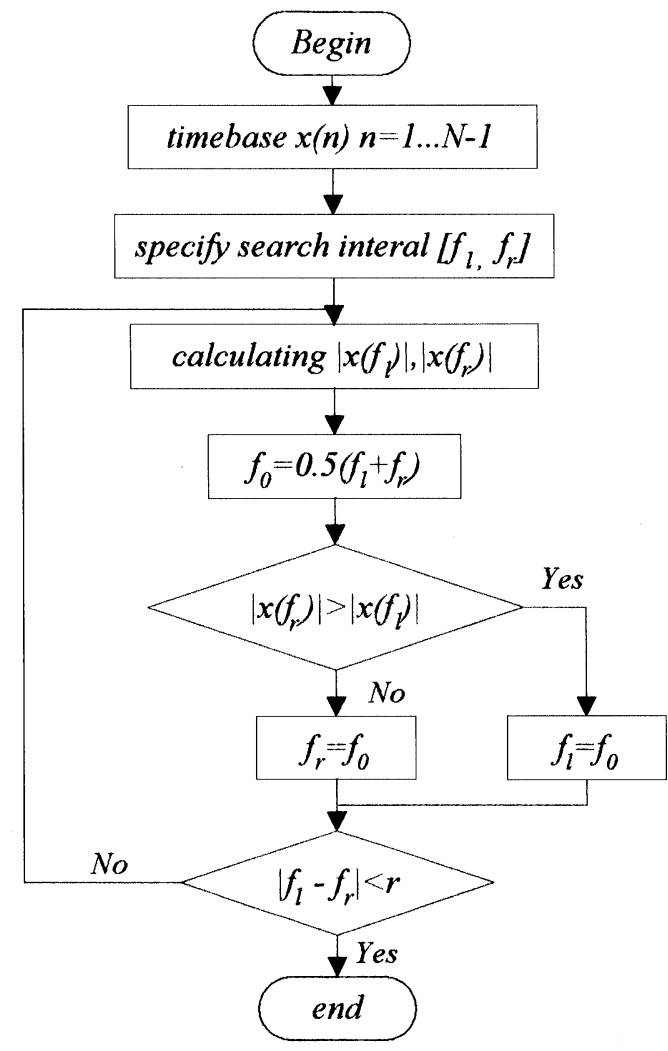

FIGURE 5 Flowchart of dichotomy search.

into (6). We can find the actual spectrum line which makes $|X(f)|$ maximum:

$$
X(f)=\frac{1}{N} \sum_{n=0}^{N-1} x(n) \mathrm{e}^{-\mathrm{j} 2 \pi n f / f_{\mathrm{s}}} .
$$

IFS can be described as follows (Fig. 5). At first, we specify the frequency interval $\left[f_{1}, f_{\mathrm{r}}\right]$ in which a unique amplitude peak to search exists. In general, we set up $f_{1}=k \Delta f, f_{\mathrm{r}}=(k+1) \Delta f$. Then, we apply Eq. (5) to compute $\left|X\left(f_{1}\right)\right|$ and $\left|X\left(f_{\mathrm{r}}\right)\right|$. Finally, we compare the above two values and substitute $f_{0}=0.5\left(f_{1}+f_{\mathrm{r}}\right)$ for the smaller one. This process is repeated until $f_{\mathrm{r}}-f_{1}<r$. We could obtain actual peak value to acquaint precise amplitude, frequency and phase information according to search with dichotomy in specified interval $\left[f_{1}, f_{\mathrm{r}}\right]$. The complexity of calculation is $\mathrm{O}(N I T)$, where $I T$ is iteration number and less than 10 . 
In order to verify if this method is effective, a simulating signal $x=25 \sin (2 \pi 77.2 t+68 \pi / 180)$ is sampled at a rate of $2000 \mathrm{~Hz}$. The correction results are shown in Table I.

Figure 6 shows the course that interpolating frequency is close to actual frequency with iteration number increasing. It is obvious that the error is almost a constant when number of iterations exceed 8. On the other hand, in order to verify if this method possesses a robust property in comparison with AFFT method proposed by Renders and Schoakens (1984), we add $10 \%$ noise with the singal. Table I illustrates the results with two different correction methods. It is obvious that the accuracy of IFS is much higher than AFFT. At the same time, the result (Fig. 7) in all kinds of window lengths illustrates that frequency error is lesser than $1.5 \%$.

According to the above simulating experiment, it is proved that IFS possesses the following advantages:

(1) In comparison with some other correction method, the accuracy of IFS is higher. It is more obvious to process short data.

(2) The complexity of calculation of IFS $(\mathrm{O}(N I T))$ is nearly equal to FFT $\left(\mathrm{O}\left(N \log _{2} N\right)\right)$.

(3) This correction method is independent of the choice of window function.

(4) IFS is not sensitive to the noise and possesses a robust property.

\subsection{Selection of Optimum Window Length}

In order to improve the accuracy of transient vibration analysis in run-up stages, we have to carefully select window length according to transient signal. Theoretically, the corrected frequency error originates from frequency interval $(\Delta f)$ and rotating speed variation in run-up stages.

TABLE I The correction results for different methods

\begin{tabular}{lccccc}
\hline & Actual value & IFS & IFS (10\% noise) & AFFT & AFFT (10\% noise) \\
\hline Frequency (Hz) & 77.2000 & 77.2104 & 77.1352 & 77.2273 & 77.0937 \\
Amplitude & 25.0000 & 24.9865 & 24.9768 & 24.9775 & 24.9463 \\
Phase (deg.) & 68.0000 & 68.0001 & 69.0172 & 67.3006 & 69.5554 \\
\hline
\end{tabular}

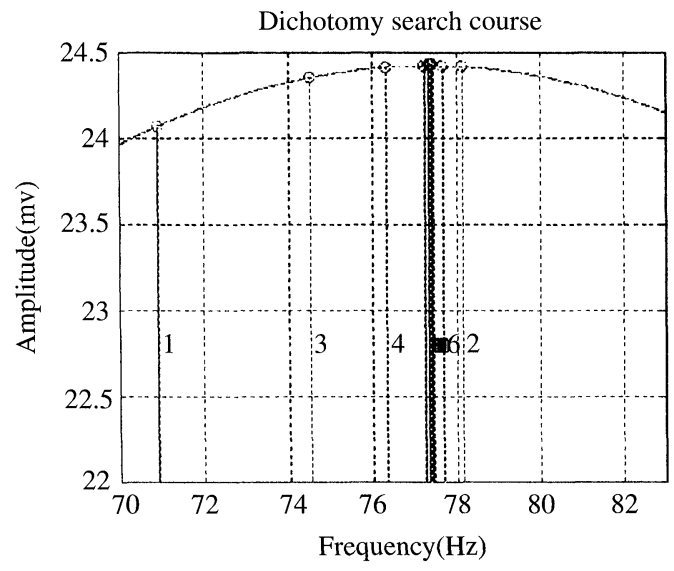

FIGURE 6 Conceptual diagram of dichotomy search. It is obvious that the error is almost a constant when the number of iterations exceed 8 .

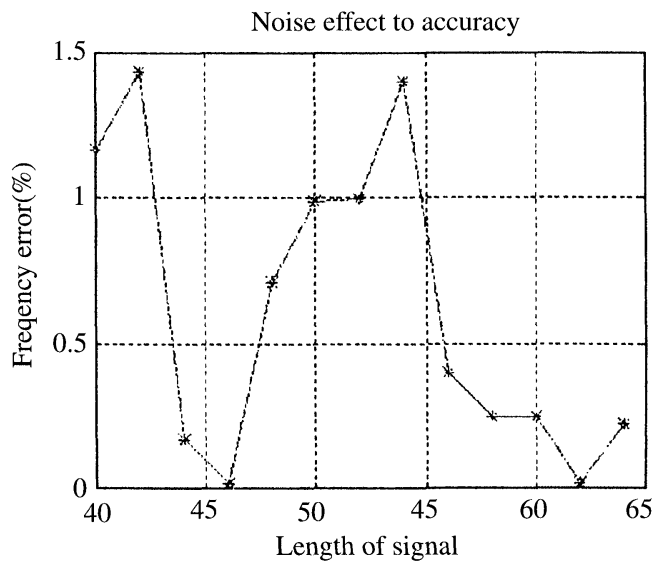

FIGURE 7 Frequency error curve with window length. The result in all kinds of window lengths illustrates that frequency error is lesser than $1.5 \%$. 
The corrected frequency error can be expressed as

$$
\begin{aligned}
E & =E_{1}+E_{2}, \\
E_{1} & =\frac{f_{\mathrm{s}}}{W_{1}} r, \\
E_{2} & =\frac{W_{1}}{N * 60} \times \Delta R,
\end{aligned}
$$

where

$f_{\mathrm{s}} \quad$ sample frequency;

$r \quad$ acceptable error of improved Fourier transform $(r<0.01)$;

$\Delta R \quad$ rotating speed variation scope within $N$ sample values;

$W_{1} \quad$ length of the window.

From Eq. (7), if window length is longer, on the one hand, $E_{1}$ is smaller, on the other hand, $E_{2}$ is bigger. It is obvious that there must exist an optimum window length $\left(W_{1}=\sqrt{60 r f_{\mathrm{s}} N / \Delta R}\right)$ to make the corrected frequency error minimum.

\subsection{Instantaneous Purified Orbit (IPO) Generation}

Figure 8 shows the flowchart to construct an IPO. First, the vibration signal $x(t)$ is multiplied with window function $\gamma(t)$ from start point $t_{0}$, then the Fourier transform of the product $x(\tau) \bar{\gamma}(t-\tau)$ is applied to $X\left(t_{0}, f\right)$. According to the above improved Fourier spectrum, the precise amplitude, frequency and initial phase of $x(\tau) \bar{\gamma}(t-\tau)$ is calculated. Then, we construct a holospectrum in this time window. By moving $\gamma(t)$ and repeating the same operation to the end of data $\left(t_{0}+\tau \geq T\right)$, the IPO is obtained. In addition, because the Hamming window possesses the border main-lobe than any other function, we select it as a translation time window.

\section{APPLICATION}

\subsection{Transient Vibration Signal in Run-up Stages}

In run-up or shutdown stages, the vibration signal is typically transient along with frequency and

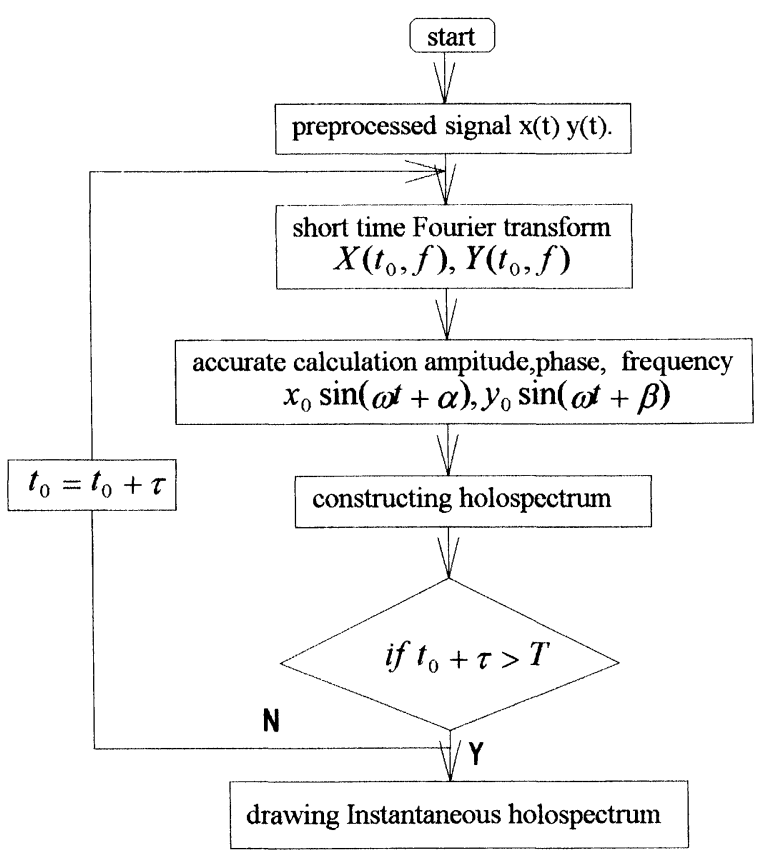

FIGURE 8 The generating procedure of IPO.

amplitude modulations owing to acceleration and the characteristics of the rotating machinery itself. Therefore, the characteristics of transient vibration cannot be precisely described by means of the conventional spectrum cascade plot. Particularly, when we have no ample hardware (such as ratio synthesizer and anti-aliasing tracking filter) to sample at a rate proportional to the shaft speed, it is difficult to analyze the transient properties of vibration in run-up stage. Fortunately, the IPO method is an effective tool which can substitute for this complex hardware in order to accomplish this analysis. The characteristics of the rotor vibration, such as the critical speed and synchronous amplification factor, can be obtained by analyzing the variation regulation of rotating frequency ellipse in a series window. In order to verify IPO as being both scientific and effective, we present the following laboratory test result.

The test rig consists of two rigid disks and two bearing supports. The rotor system is driven by an electric motor incorporated with shaft through a 
flexible coupling. Each data set consists of 1200 data points and is sampled at a rate of $1000 \mathrm{~Hz}$. According to Eq. (6), the optimum window length is 48 points.

From the orbit diagram (Fig. 9), there is no means to analyze vibration characteristics clearly. Regular FFT spectrum (Fig. 10) gives us an average effect and conceals the transient property of the rotor in passing through a critical speed. Meanwhile, SPFT (Figs. 11 and 12), whose time length is 48 , illustrates how vibration amplitude varies with time as well as frequency axis to some extent. However, owing to lower resolution along

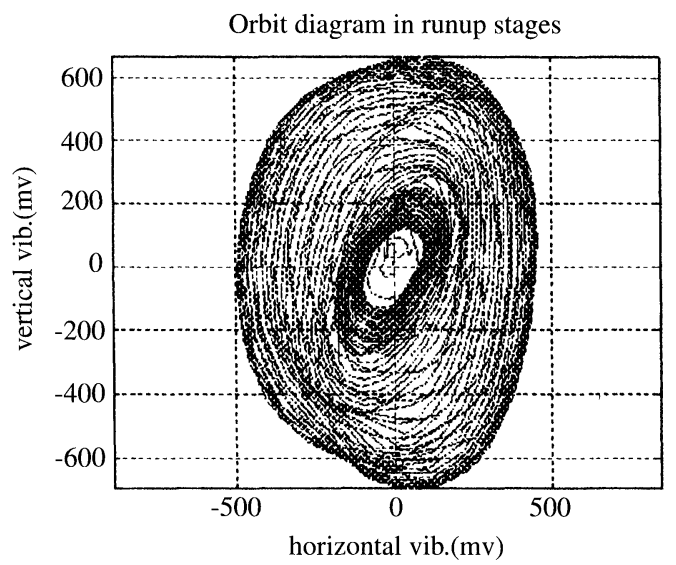

FIGURE 9 Orbit diagram in run-up stages. From the orbit diagram, there is no means to analyze vibration characteristics clearly.
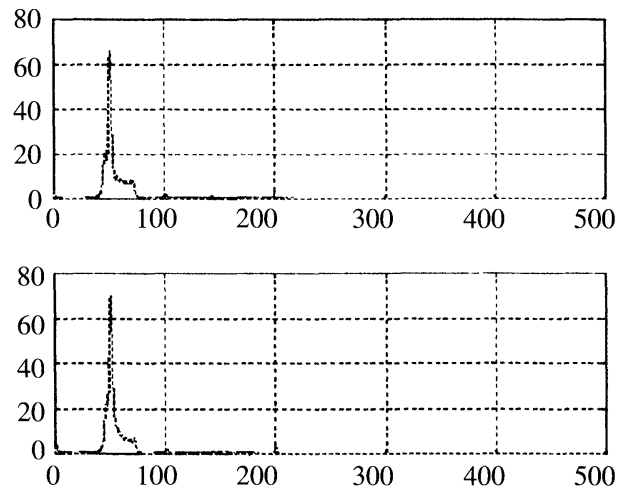

FIGURE 10 FFT spectra in run-up stages. Regular FFT spectrum gives us an average effect and conceals the transient property of the rotor in passing through a critical speed. frequency axis, the frequency-varying property is almost concealed.

Fortunately, IPO gives us a panorama of rotor vibration accurately when passing through critical speed. In this circumstance, precession direction of the rotor is entirely forward owing to isotropic properties of the supports. It is confirmed clearly by IPO from Fig. 13. Except for precession direction, the other properties such as inclination angle of major axis, vibration power and eccentricity of rotating-frequency ellipse are manifested completely. From Fig. 13, the vibration power and of rotating frequency component gradually increases

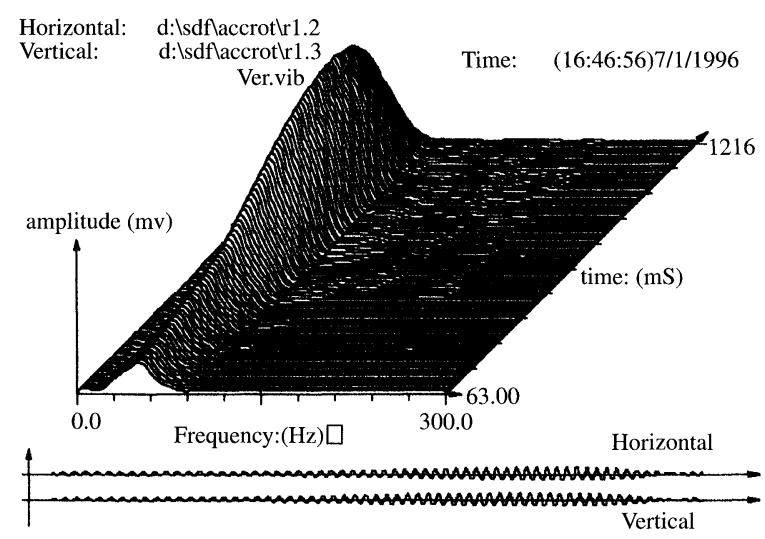

FIGURE 11 SPFT of horizontal vibration.

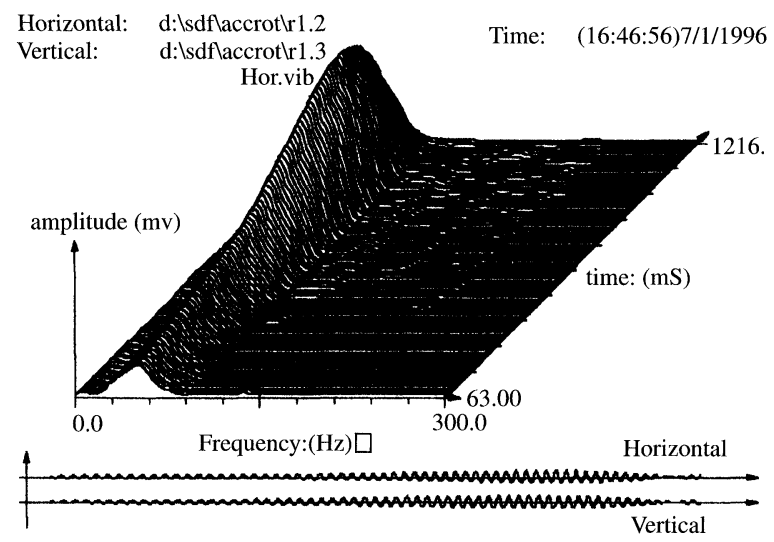

FIGURE 12 SPFT of vertical vibration. 


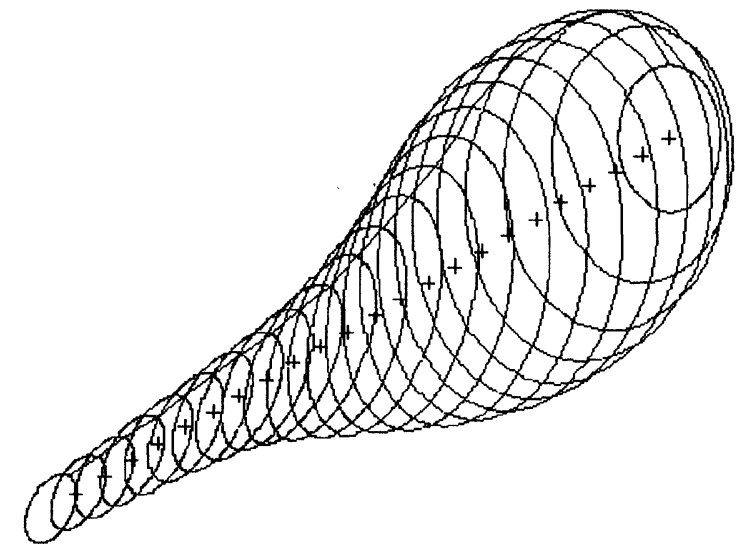

FIGURE 13 Instantaneous purified orbit in run-up stages (positive precession direction of rotor is denoted by ' + '). IPO gives us a panorama of rotor vibration accurately when passing through critical speed.

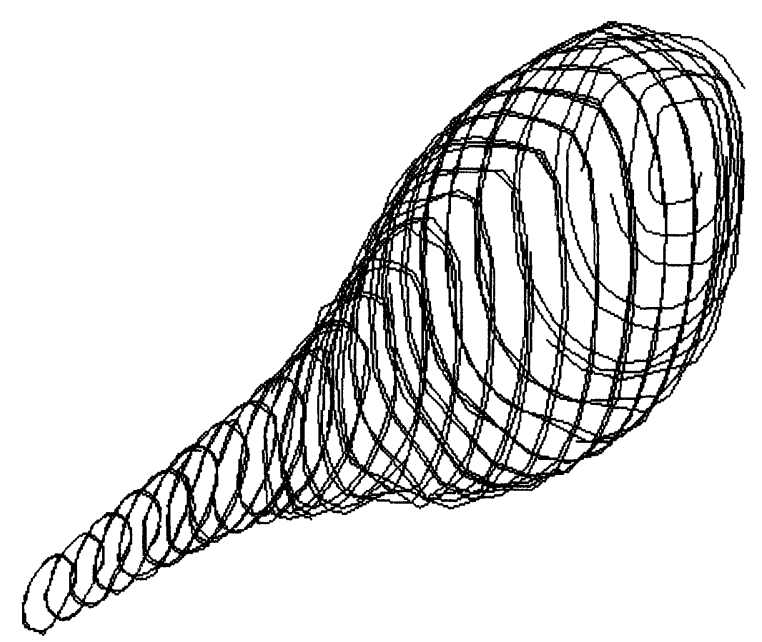

FIGURE 14 Instantaneous orbit in run-up stages. In comparison with IPO, two diagrams are very similar to each other.

from 1st window to 20th window, and then suddenly decreases. It is obvious that the point of critical speed is located in the 20th window.

In order to check whether the frequency, amplitude and phase of instantaneous harmonic components are accurately estimated or not, original orbit diagram is presented, with same window length along the time axis, in Fig. 14. In comparison with IPO, the two diagrams are very similar to each

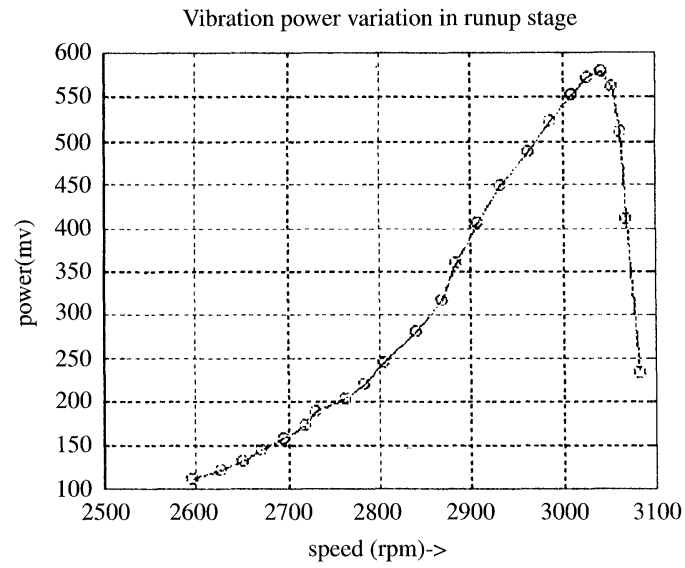

FIGURE 15 Amplitude-frequency property curve. According to amplitude-frequency property curve, we can specify that the critical speed of rotor system is $3042 \mathrm{rpm}$.

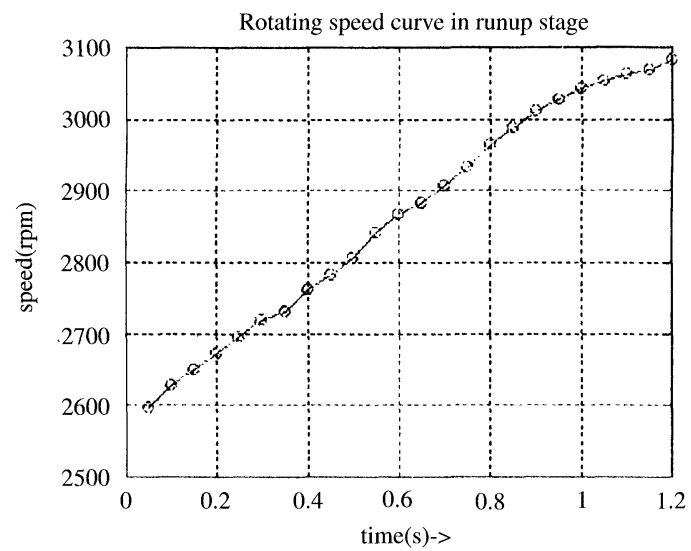

FIGURE 16 Rotating speed curve in run-up stages. It illustrates the course of rotating speed accelerating in run-up stages.

other. It is proved that the magnitudes of harmonic components are correct, and IPO is effective and accurate.

In order to analyze the time-varying and frequency-varying properties, we plot the amplitude-frequency property curve and rotating speed curve by means of IPO. According to amplitudefrequency property curve (Fig. 15), we can specify that the critical speed of rotor system is $3042 \mathrm{rpm}$. It is very close to the designed critical speed. Meanwhile, Fig. 16 illustrates the course of rotating speed accelerating in run-up stages. Obviously, 
the rotor was accelerated from 2600 to $3100 \mathrm{rpm}$ within $1.2 \mathrm{~s}$.

\subsection{Bistable Vibration Condition}

In general, large rotating machinery runs in a stable condition. However, its orbit sometimes is composed of two limit cycles. It runs in one state steadily at first, and then, jumps from the current state to another. It is difficult to accurately describe such behavior by means of the traditional methods. Fortunately, the IPO method can show such bistable phenomenon of rotor systems. From an FFT spectrum (Fig. 17), the rotor running in bistable condition shows no difference from that in normal condition. On account of the average effect, the bistable feature is concealed in FFT spectrum. In orbit diagram (Fig. 18), in spite of the existing two cycles, we cannot be certain whether the machine is running in a bistable condition or not.

From IPO (Fig. 19), we can find the following characteristics of rotor.

(1) Precession direction From the 1st window to 7 th window of vibration signal, rotor has negative precession. And then, the rotor has positive precession from the 7 th window to the end.

(2) Inclination angle of major $\phi$ It is obvious that the inclination angle of major axis jumps from
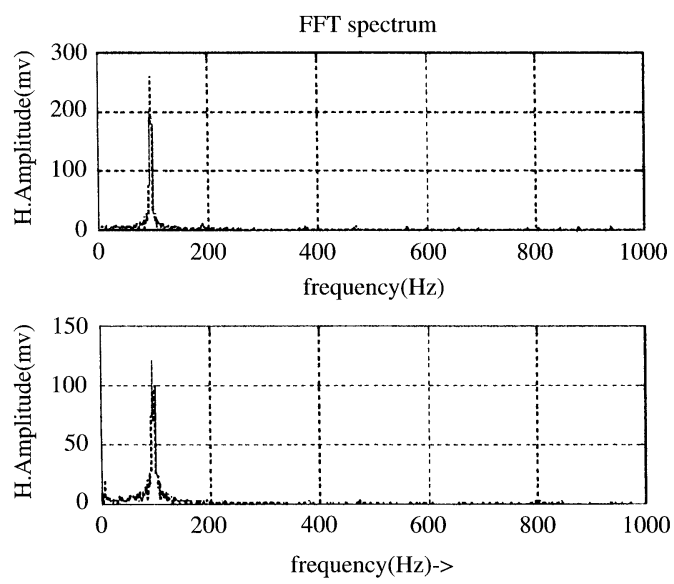

FIGURE 17 Orbit diagram of bistable vibration.

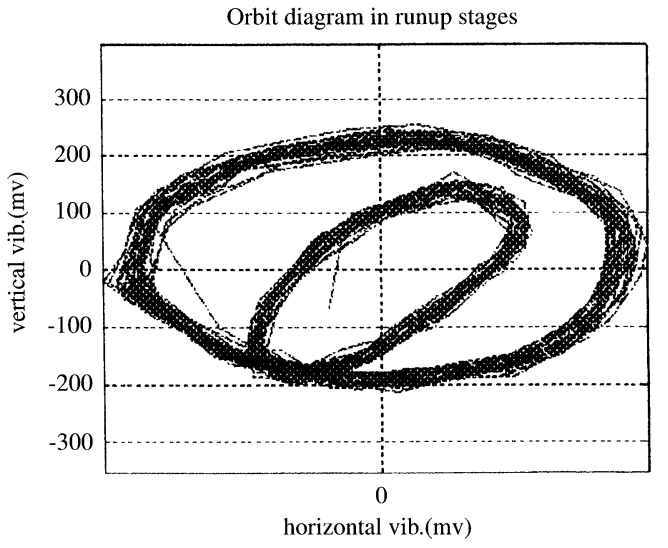

FIGURE 18 FFT spectra of bistable vibration.

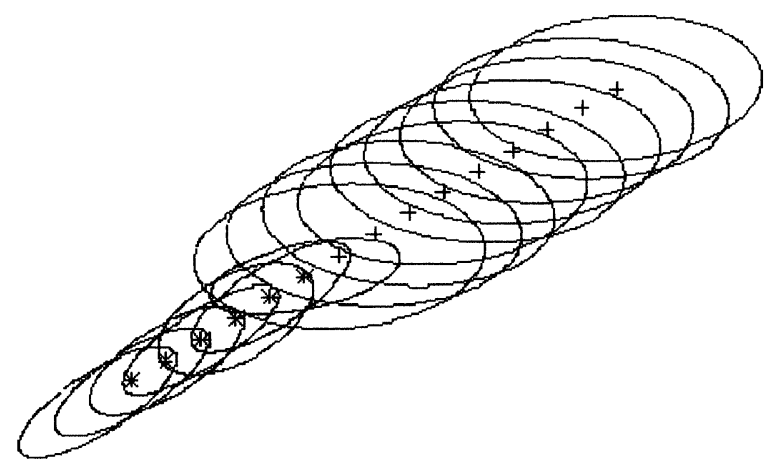

FIGURE 19 Instantaneous purified orbit of bistable vibration (positive precession direction is denoted by ' + ' negative precession direction is denoted by ' $*$ ').

$45^{\circ}$ to $20^{\circ}$ at 7 th time window. However, it is very stable in any other window.

(3) Vibration power Obviously, it increases in 7th time window immediately.

\section{CONCLUSION}

This work aims at studying the drawbacks of traditional analysis methods. The IPO based on an improved Fourier transform based on combination of SPFT and holospectrum is presented in this paper. In comparison with the above two methods, it shows a panorama of orbit when the rotor is running in unstable condition. Consequently, we 
can accurately analyze the transient behavior of rotor vibration by means of rotating frequency ellipse variation in a series of time windows. Practical examples, such as transient vibration in run-up or shutdown stages, bistable condition of rotor, show that IPO is a powerful tool to diagnose and analyze the vibration behavior of a rotor system. The behavior of rotating-frequency ellipse parameters when the rotor passes through the critical speed is very attractive and worth further research.

\section{NOMENCLATURE}

\begin{tabular}{|c|c|}
\hline FFT & Fast Fourier transform \\
\hline IFS & Improved Fourier spectrum \\
\hline SPFT & Short period Fourier transform \\
\hline IPO & Instantaneous purified orbit \\
\hline$x(t), y(t)$ & $\begin{array}{l}\text { Rotor lateral vibration in two } \\
\text { orthogonal directions }\end{array}$ \\
\hline$x_{0}, w(f), \alpha$ & $\begin{array}{l}\text { Precise amplitude, frequency } \\
\text { and phase of } x \text {-direction }\end{array}$ \\
\hline$y_{0}, w(f), \beta$ & $\begin{array}{l}\text { Precise amplitude, frequency } \\
\text { and phase of } y \text {-direction }\end{array}$ \\
\hline$f_{\mathrm{e}}, A_{\mathrm{e}}, \alpha_{\mathrm{e}}$ & $\begin{array}{l}\text { The error of frequency, } \\
\text { amplitude and phase for FFT }\end{array}$ \\
\hline$X(f), Y(f)$ & $\begin{array}{l}\text { Fourier coefficient associated } \\
\text { with } x \text {-, } y \text {-direction }\end{array}$ \\
\hline$W(f)$ & $\begin{array}{l}\text { Fourier transform of window } \\
\text { function }\end{array}$ \\
\hline$X\left(t_{0}, f\right), Y\left(t_{0}, f\right)$ & SPFT of $x(t), y(t)$ \\
\hline $2 a, 2 b$ & Major, minor axes of ellipse \\
\hline$\phi$ & $\begin{array}{l}\text { Inclination angle of major axis } \\
\text { of ellipse }\end{array}$ \\
\hline$\theta$ & Initial phase of ellipse \\
\hline$f_{\mathrm{s}}$ & Sample frequency \\
\hline$W_{1}(\tau)$ & $\begin{array}{l}\text { Window length of SPFT } \\
\left(\tau=W_{\mathrm{l}} / f_{\mathrm{s}}\right)\end{array}$ \\
\hline$N(T)$ & Length of sample $\left(T=N / f_{\mathrm{s}}\right)$. \\
\hline
\end{tabular}

\section{References}

Chen, Y., Du, R. and Qu, L.S. (1995) Fault features of large machinery and diagnosis using sensor fusion. J. Sound and Vibration, 188, 227-242.

Don Southwick (1993) Using full spectrum plots. Orbit, 14(4), 12-16.

Don Southwick (1994) Using full spectrum plots, Part 2. Orbit, 15(2), 10-16.

Genta, G. and Delprete, C. (1995) Acceleration through critical speeds of an anisotropic, non-linear, torsionally stiff rotor with many degrees of freedom. J. Sound and Vibration, 180(3), 369-386.

Grankle, T. (1983) Interpolation algorithms for discrete Fourier transform of weighted signals. IEEE trans. on IM, 32(3), 350-355.

Jian Meng (1996) Some advanced techniques in fault feature extraction for large rotating machinery. Ph.D. dissertation, Xian JiaoTong University.

Lee, C.W. and Han, Y.S. (1998) Use of directional Wigner distribution for identification of the instantaneous whirling orbit in rotating machinery. 7 th ISROMAC, pp. 22-26.

Muszynska, A. (1995) Vibrational diagnostics of rotating machinery malfunctions. Int. J. of Rotating Machinery, 1(3), 237-266.

Qian, S. and Chen, D. (1996) Joint Time-Frequency Analysis. Prentice Hall.

Qingfeng Meng and Liangsheng Qu (1992) Rotating machinery faults diagnosis using Wigner distribution. Mechanical System and Signal Processing, 5(3), 155-166.

Qu, L., Liu, X. and Chen, Y. (1989) Discovering the holospectrum. J. Noise and Vibration Worldwide, 58-62, February.

Qu, L., Xie, A.X. and Li, X. (1993) Study and performance evaluation of some nonlinear diagnostic methods for large rotating machinery, Mech. Math. Theory, 28(5), 699-713.

Ramirez, R.W. (1985) The FFT: Fundamentals and Concepts. Prentice-Hall.

Renders, H. and Schoakens, J. (1984) High-accuracy spectrum analysis of sampled discrete frequency signals by analytical leakage compensation. IEEE Trans. on IM, 33(4), 287-292.

Shi, D.F. (1998) Study on Holodiagnosis for large rotating machinery. Doctor's thesis, Xian Jiaotong University.

Zheng, G.T. (1998) Vibration of a rotor system with a switching crack and detection of the crack. ASME Trans. on J. of Engineering for Gas Turbines and Power, 120(1), 149-154, 1998. 

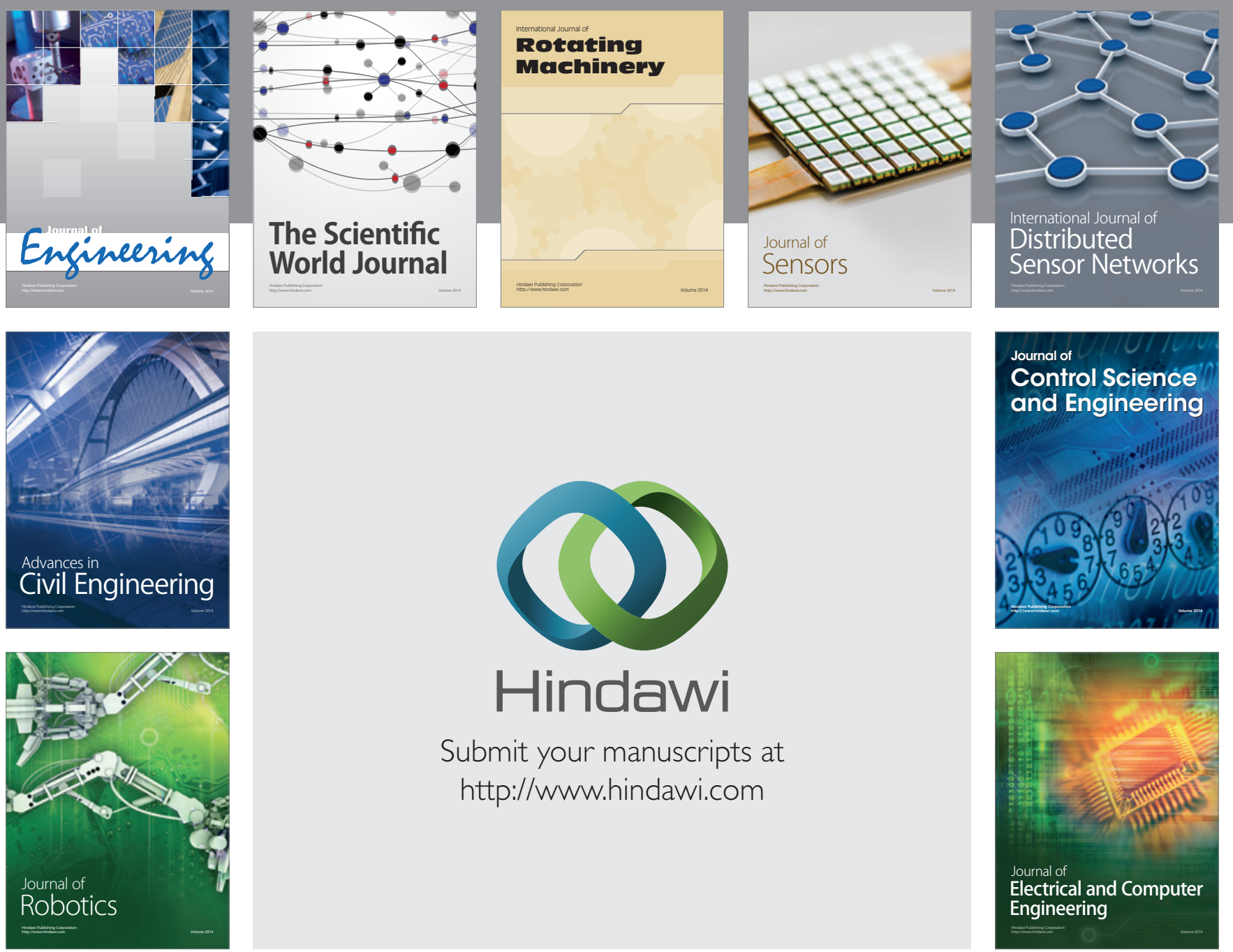

Submit your manuscripts at

http://www.hindawi.com
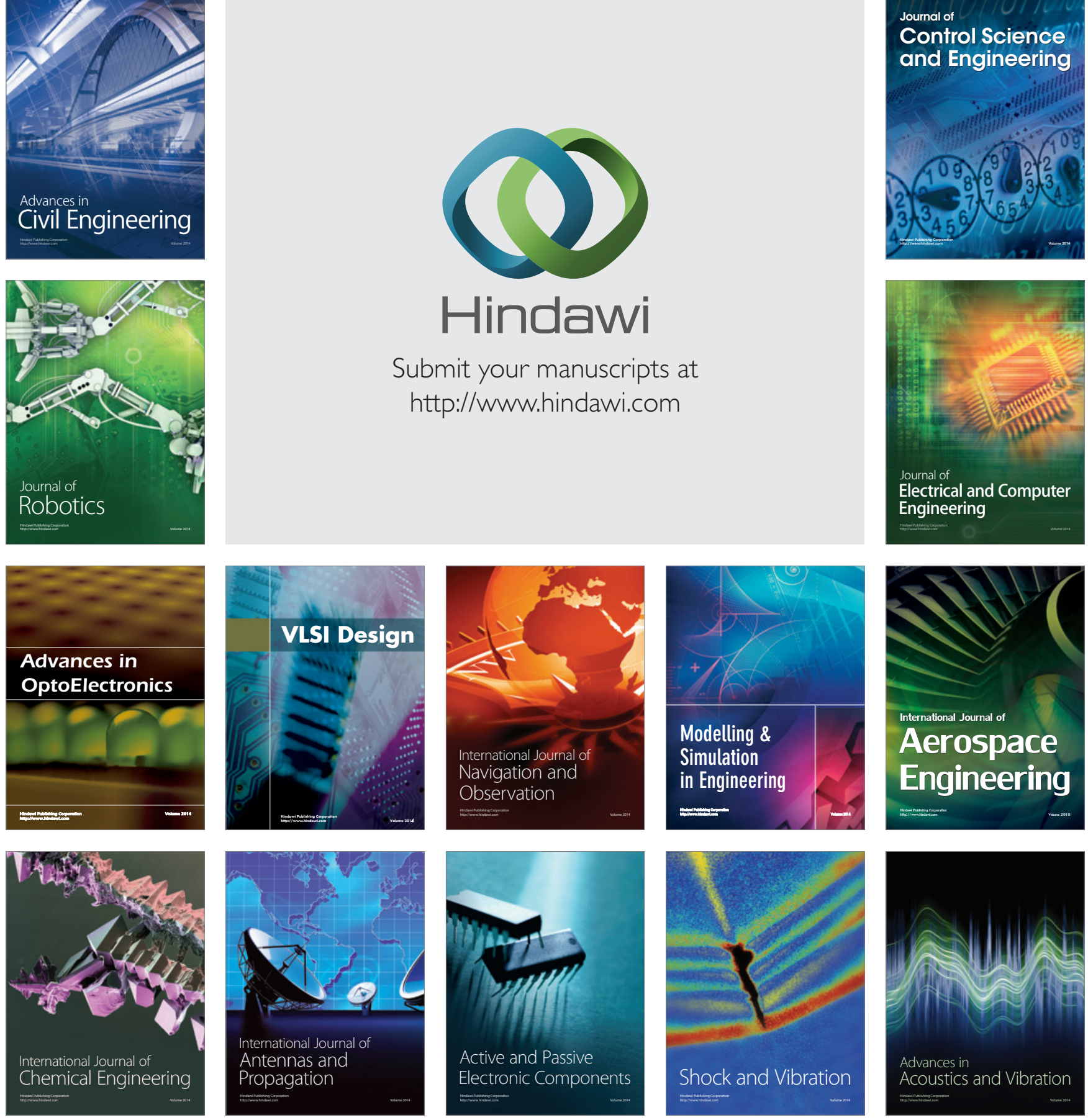